\title{
From neutron stars to quark stars in mimetic gravity
}

\author{
Artyom V. Astashenok ${ }^{1}$ and Sergei D. Odintsov ${ }^{2,3}$ \\ ${ }^{1}$ I. Kant Baltic Federal University, \\ Institute of Physics and Technology, \\ Nevskogo st. 14, 236041 Kaliningrad, Russia, \\ ${ }^{2}$ Instituciò Catalana de Recerca $i$ Estudis Avançats (ICREA), Barcelona, Spain \\ ${ }^{3}$ Institut de Ciencies de l'Espai (IEEC-CSIC), \\ Campus UAB, Facultat de Ciencies, Torre C5-Par-2a pl, \\ E-08193 Bellaterra, Barcelona, Spain
}

\begin{abstract}
Realistic models of neutron and quark stars in the framework of mimetic gravity with Lagrange multiplier constraint are presented. We discuss the effect of mimetic scalar aiming to describe dark matter on mass-radius relation and the moment of inertia for slowly rotating relativistic stars. The mass-radius relation and moment of inertia depend on the value of mimetic scalar in the center of star. This fact leads to the ambiguity in the mass-radius relation for a given equation of state. Such ambiguity allows to explain some observational facts better than in standard General Relativity. The case of two mimetic potentials namely $V(\phi) \sim A \phi^{-2}$ and $V(\phi) \sim A e^{B \phi^{2}}$ is considered in detail. The relative deviation of maximal moment of inertia is approximately twice larger than the relative deviation of maximal stellar mass. We also briefly discuss the mimetic $f(R)$ gravity. In the case of $f(R)=R+a R^{2}$ mimetic gravity it is expected that increase of maximal mass and maximal moment of inertia due to mimetic scalar becomes much stronger with bigger parameter $a$. The contribution of scalar field in mimetic gravity can lead to possible existence of extreme neutron stars with large masses.
\end{abstract}

Keywords: mimetic gravity; neutron stars; quark stars.

\section{INTRODUCTION}

Number of modified gravity theories which may describe accelerating universe has been intensively studied in the recent years (for a review, see [1]). In the framework of these theories it is possible to obtain the accelerated expansion of Universe [2 4] without using of inflaton for inflation and/or scalars, fluids or cosmological constant for dark energy. Some cosmological bounds also favour the modified gravity [1]. However, one can not discriminate between the $\Lambda$ CDM model or the modified gravity using only cosmological bounds.

The study of alternative gravities on the astrophysical level, e.g. using the relativistic stars, allows for an alternative way of discrimination between General Relativity (GR) from its possible modifications [5]. The fundamental question is the existence of stable neutron stars in given $f(R)$ theory [6, 7]. Secondly, one needs to compare the mass-radius relation, moment of inertia, quadrupole moment, Love number and other relevant characteristics of stars in GR and $f(R)$ gravity [8]. Finally, it is interesting to consider the possible emergence of new stellar structures in modified gravities (stable stars with large central densities or/and with large magnetic fields, (super)massive stars etc.). The discovery of such structures will constitute a powerful signature for the Extended Gravity [9, 10]. The structure of compact stars in perturbative $f(R)$ gravity was investigated recently in refs. 11 13]. In this approach the scalar curvature $R$ is defined by Einstein equations at zeroth order on the small parameter, i.e. $R \sim T$, where $T$ is the trace of energy-momentum tensor. Non-perturbative studies are also available for non-rotating, slowly and fast rotating compact star models [14, 15].

In this paper we investigate relativistic stars in mimetic gravity with scalar potential $V(\phi)$ (mimetic potential) and with Lagrange multiplier $\beta(\phi)$. This theory was recently proposed in ref. [17] for eventual geometric description of dark matter. The paper is organized as follows. In the next section, we present the field equations for mimetic $f(R)$ gravity with mimetic scalar potential. For spherically symmetric solutions of these equations, the modified Tolman-Oppenheimer-Volkoff (TOV) equations are derived. The corresponding scalar-tensor description of mimetic $f(R)$ theory is given in the third section. Mimetic $f(R)$ theory is equivalent to a two scalar-tensor gravity. In Section IV, the compact star models in frame of simple mimetic gravity with $f(R)=R$ are investigated in detail. We obtain the mass-radius and mass-moment of inertia relations for neutron stars in the case of simple mimetic potentials. For neutron stars we use a well-known equation of state proposed by Douchin and Haensel (Sly4 EoS) [16]. We also investigate the case of quark stars with simple EoS for deconfined quark matter. Some conclusions are given in Summary. 


\section{MODIFIED TOV EQUATIONS IN MIMETIC $f(R)$ GRAVITY WITH SCALAR POTENTIAL}

The main feature of mimetic approach to gravity is that the conformal symmetry is a non-violated internal degree of freedom. The physical metric $g_{\mu \nu}$ can be represented via so-called auxiliary metric as [17]

$$
g_{\mu \nu}=-\hat{g}^{\rho \sigma} \partial_{\rho} \phi \partial_{\sigma} \phi \hat{g}_{\mu \nu}
$$

Here $\phi$ and $\hat{g}_{\mu \nu}$ are mimetic field and an auxiliary metric tensor correspondingly. Then one takes variation with respect to the auxiliary metric $\hat{g}_{\mu \nu}$ and to the scalar field $\phi$ instead of the physical metric. This variation gives the following condition on auxiliary scalar field $\phi$ :

$$
g^{\rho \sigma}\left(\hat{g}_{\mu \nu}, \phi\right) \partial_{\rho} \phi \partial_{\sigma} \phi=-1
$$

Such parametrization is invariant under Weyl transformations $\hat{g}_{\mu \nu}=e^{\sigma(x)} g_{\mu \nu}$ and therefore the auxiliary metric tensor doesn't appear in the action for the gravitational field.

For spherically symmetric solution describing the star one needs to consider a metric of the following form

$$
d s^{2}=-e^{2 \psi} d t^{2}+e^{2 \lambda} d r^{2}+r^{2} d \Omega^{2} .
$$

The metric functions $\psi$ and $\lambda$ depend only from radial coordinate. In addition, we assume that the auxiliary scalar field depends only on the $r$. The action for mimetic $f(R)$ theory with a scalar potential $V(\phi)$ and a Lagrange multiplier $\beta$ in the Jordan frame (in units where $G=c=1$ ) is given as [18]:

$$
S=\frac{1}{16 \pi} \int d^{4} x \sqrt{-g}\left[f\left(R\left(g_{\mu \nu}\right)-V(\phi)+\beta\left(g^{\mu \nu} \partial_{\mu} \phi \partial_{\nu} \phi+1\right)\right]+S_{\text {matter }}\right.
$$

where $g$ is determinant of the metric $g_{\mu \nu}$ and $S_{\text {matter }}$ is the action of the standard perfect fluid matter. Note that above theory may consistently unify the early-time inflation with late-time acceleration and geometric dark matter 18]. The accelerating cosmology of such mimetic gravity was investigated recently in refs. [19]. It is also interesting that mimetic scalar may eventually play the role of chameleon [20].

Varying the action with respect to $g_{\mu \nu}$ gives us the equation of motion for metric functions:

$$
\begin{aligned}
f^{\prime}(R) G_{\mu \nu}-\frac{1}{2}\left(f(R)-f^{\prime}(R) R\right) g_{\mu \nu} & -\left(\nabla_{\mu} \nabla_{\nu}-g_{\mu \nu} \square\right) f^{\prime}(R) \\
& \left.=8 \pi T_{\mu \nu}+g_{\mu \nu}\left(-V(\phi)+\beta\left(g^{\rho \sigma} \partial_{\rho} \phi \partial_{\sigma} \phi+1\right)\right)-2 \beta \partial_{\mu} \phi \partial_{\nu} \phi\right) .
\end{aligned}
$$

Here $G_{\mu \nu}=R_{\mu \nu}-\frac{1}{2} R g_{\mu \nu}$ is the Einstein tensor, $f^{\prime}(R)=d f(R) / d R$ is the derivative of function $f(R)$ with respect to the scalar curvature $R$ and $T_{\mu \nu}$ is the energy-momentum tensor. For perfect fluid we have $T_{\mu \nu}=$ $\operatorname{diag}\left(e^{2 \psi} \rho, e^{2 \lambda} p, r^{2} p, r^{2} \sin ^{2} \theta p\right)$, where $\rho$ is the matter density and $p$ is the pressure.

Assuming that $\phi=\phi(r)$ and varying the action with respect to $\beta$ one can obtain the following constraint for the auxiliary field:

$$
e^{-2 \lambda}\left(\frac{d \phi}{d r}\right)^{2}+1=0
$$

One may consider substituting $\phi$ as follows $\phi \rightarrow \phi^{*}=i \phi$. Therefore we have the following equation for $\phi^{*}$ :

$$
e^{-2 \lambda}\left(\frac{d \phi^{*}}{d r}\right)^{2}=1
$$

which in the limit $r \rightarrow \infty$ becomes $\left(\frac{d \phi^{*}}{d r}\right)^{2} \rightarrow 1$.

The Tolmann-Oppenheimer-Volkov (TOV) equations for this theory of gravity are nothing else that " $t t^{\prime}$ and " $r r$ " components of the field equations (5):

$$
\begin{aligned}
\frac{f^{\prime}(R)}{r^{2}} \frac{d}{d r}\left[r\left(1-e^{-2 \lambda}\right)\right] & =8 \pi \rho+V(\phi)++\frac{1}{2}\left(f^{\prime}(R) R-f(R)\right) \\
& +e^{-2 \lambda}\left[\left(\frac{2}{r}-\frac{d \lambda}{d r}\right) \frac{d f^{\prime}(R)}{d r}+\frac{d^{2} f^{\prime}(R)}{d r^{2}}\right]
\end{aligned}
$$




$$
\begin{aligned}
\frac{f^{\prime}(R)}{r}\left[2 e^{-2 \lambda} \frac{d \psi}{d r}-\frac{1}{r}\left(1-e^{-2 \lambda}\right)\right] & =8 \pi p-V(\phi)-\beta\left(e^{-2 \lambda}\left(\frac{d \phi}{d r}\right)^{2}-1\right)+ \\
& +\frac{1}{2}\left(f^{\prime}(R) R-f(R)\right)+e^{-2 \lambda}\left(\frac{2}{r}+\frac{d \psi}{d r}\right) \frac{d f^{\prime}(R)}{d r}
\end{aligned}
$$

For the scalar $\phi$ we have equation of motion

$$
2 \nabla^{\mu}\left(\beta \partial_{\mu} \phi\right)+\frac{d V(\phi)}{d \phi}=0 .
$$

The hydrostatic equilibrium conditionfollows from the conservation law, $\nabla^{\mu} T_{\mu \nu}=0$ i.e.

$$
\frac{d p}{d r}=-(\rho+p) \frac{d \psi}{d r}
$$

In $\mathrm{f}(\mathrm{R})$ gravity the scalar curvature is dynamical variable and an equation for $R$ can be obtained using the trace of field equation (5]). One gets

$$
3 \square f^{\prime}(R)+f^{\prime}(R) R-2 f(R)=-8 \pi(\rho-3 p)-4 V(\phi)+2 \beta,
$$

where

$$
e^{2 \lambda} \square=\left(\frac{2}{r}+\frac{d \psi}{d r}-\frac{d \lambda}{d r}\right) \frac{d}{d r}+\frac{d^{2}}{d r^{2}} .
$$

For $f(R)=R$ this equation is reduced to the form

$$
R=8 \pi(\rho-3 p)+4 V(\phi)-2 \beta
$$

Inside the star the equations (8), (9), (11), (12) can be solved numerically for a given equation of state (EOS) $p=f(\rho)$ and boundary conditions $\lambda(0)=0, R(0)=R_{c}, R^{\prime}(0)=0$ and $\rho(0)=\rho_{c}$.

Outside the star $(\rho=p=0)$ the solution is defined by the Eqs. (8), (9), (12), while on the surface of star $\left(r=r_{s}\right)$ the junction conditions should be satisfied:

$$
\lambda_{\text {in }}\left(r_{s}\right)=\lambda_{\text {out }}\left(r_{s}\right), \quad R_{\text {in }}\left(r_{s}\right)=R_{\text {out }}\left(r_{s}\right), \quad R_{\text {in }}^{\prime}\left(r_{s}\right)=R_{\text {out }}^{\prime}\left(r_{s}\right)
$$

The gravitational mass parameter $m(r)$ is defined as

$$
e^{-2 \lambda}=1-\frac{2 m}{r} .
$$

Finally, the asymptotic flatness requirement gives the constraints on scalar curvature and mass parameter:

$$
\lim _{r \rightarrow \infty} R(r)=0, \lim _{r \rightarrow \infty} m(r)=\text { const. }
$$

\section{SCALAR-TENSOR DESCRIPTION FOR MIMETIC $f(R)$ GRAVITY}

By analogy with convenient $F(R)$ one can consider mimetic $f(R)$ theory in the Einstein frame. In this case the theory is just a two scalar-tensor gravity. We start from the equivalent Brans-Dicke action:

$$
S_{g}=\frac{1}{16 \pi} \int d^{4} x \sqrt{-g}\left(\Phi R-U(\Phi)-V(\phi)+\beta(\phi)\left(g^{\mu \nu} \partial_{\mu} \phi \partial_{\nu} \phi+1\right)\right)
$$

Here $\Phi=d f(R) / d R$ is gravitational scalar and $U(\Phi)=R f^{\prime}(R)-f(R)$ is a potential. Using conformal transformation $\tilde{g}_{\mu \nu}=\Phi g_{\mu \nu}$ one can write the action in the Einstein frame as follows:

$$
S_{g}=\frac{1}{16 \pi} \int d^{4} x \sqrt{-\tilde{g}}\left(\tilde{R}-2 \tilde{g}^{\mu \nu} \partial_{\mu} \varphi \partial_{\nu} \varphi-4 \tilde{V}(\varphi)-V(\phi) e^{-4 \varphi / \sqrt{3}}+\beta(\phi) e^{-2 \varphi / \sqrt{3}}\left(\tilde{g}^{\mu \nu} \partial_{\mu} \phi \partial_{\nu} \phi+e^{-2 \varphi / \sqrt{3}}\right)\right)
$$


where $\varphi=\frac{\sqrt{3}}{2} \ln \Phi$ and the redefined potential $V(\varphi)$ in Einstein frame becomes $\tilde{V}(\varphi)=\Phi^{-2}(\varphi) U(\Phi(\varphi)) / 4$.

The form of the spacetime metric can be chosen to coincide formally with the GR form (3):

$$
d \tilde{s}^{2}=\Phi d s^{2}=-e^{2 \tilde{\psi}} d t^{2}+e^{2 \tilde{\phi}} \tilde{d r}^{2}+\tilde{r}^{2} d \Omega^{2} .
$$

In Eq. (17) $\tilde{r}^{2}=\Phi r^{2}, e^{2 \tilde{\psi}}=\Phi e^{2 \psi}$ and from the equality $\Phi e^{2 \lambda} d r^{2}=e^{2 \tilde{\lambda}} d \tilde{r}^{2}$ follows that

$$
e^{-2 \lambda}=e^{-2 \tilde{\lambda}}\left(1-\tilde{r} \varphi^{\prime}(\tilde{r}) / \sqrt{3}\right)^{2}
$$

Therefore the mass parameter $m(r)$ can be obtained from $\tilde{m}(\tilde{r})$ and gets the form:

$$
m(\tilde{r})=\frac{\tilde{r}}{2}\left(1-\left(1-\frac{2 \tilde{m}}{\tilde{r}}\right)\left(1-\tilde{r} \varphi^{\prime}(\tilde{r}) / \sqrt{3}\right)^{2}\right) e^{-\varphi / \sqrt{3}}
$$

The resulting equations for metric functions $\tilde{\lambda}$ and $\tilde{\psi}$ coincide in fact with ordinary TOV equations for mimetic General Relativity in which the energy density and pressure of the scalar field $\varphi$ are included (the tildes are omitted for simplicity):

$$
\begin{aligned}
\frac{1}{r^{2}} \frac{d}{d r}\left[r\left(1-e^{-2 \lambda}\right)\right] & =e^{-4 \varphi / \sqrt{3}}(8 \pi \rho+V(\phi))+e^{-2 \lambda}\left(\frac{d \varphi}{d r}\right)^{2}+\tilde{V}(\varphi), \\
\frac{1}{r}\left[2 e^{-2 \lambda} \frac{d \psi}{d r}-\frac{1}{r}\left(1-e^{-2 \lambda}\right)\right] & =e^{-4 \varphi / \sqrt{3}}\left(8 \pi p-\beta(\phi)\left(e^{-2 \lambda} e^{2 \varphi / \sqrt{3}}\left(\frac{d \phi}{d r}\right)^{2}-1\right)\right)+e^{-2 \lambda}\left(\frac{d \varphi}{d r}\right)^{2}-\tilde{V}(\varphi) .
\end{aligned}
$$

The hydrostatic equilibrium condition is written as

$$
\frac{d p}{d r}=-(\rho+p)\left(\frac{d \psi}{d r}-\frac{1}{\sqrt{3}} \frac{d \varphi}{d r}\right) .
$$

The equation (10) for the mimetic field becomes:

$$
2 \nabla^{\mu}\left(\beta e^{-2 \varphi / \sqrt{3}} \partial_{\mu} \phi\right)+V^{\prime}(\phi) e^{-4 \varphi / \sqrt{3}}=0 .
$$

Finally, the last equation of motion for the scalar field is equivalent to Eq. (12) in $f(R)$ theory:

$$
\square \varphi+\frac{d V(\varphi)}{d \varphi}=-\frac{4 \pi}{\sqrt{3}} e^{-4 \varphi / \sqrt{3}}(\rho-3 p)-\frac{1}{\sqrt{3}} e^{-4 \varphi / \sqrt{3}}(2 V(\phi)-\beta) \text {. }
$$

The above formulation maybe used to study relativistic stars in the Einstein frame. Unfortunately, due to number of issues (the appearence of singular points where mathematical equivalence is lost, the issue of boundary conditions in different frames, wider space of physical variables(negative values of scalar)) the physical equivalence with Jordan frame may not be explicit. To avoid all above problems, eventually it is better to work with $F(R)$ frame.

\section{RELATIVISTIC STARS IN MIMETIC GENERAL RELATIVITY}

Let us study models of relativistic compact stars in mimetic General Relativity. Even in this case some interesting deviations from convenient GR can be observed.

\section{A. Neutron stars}

There are many equations of state for dense nuclear matter (see for example [21, 22]). One of the most commonly used is the SLy4 EOS. Here we use an analytic fitting of the EOS, see [21]:

$$
\begin{aligned}
\zeta & =\frac{a_{1}+a_{2} \xi+a_{3} \xi^{3}}{1+a_{4} \xi} f\left(a_{5}\left(\xi-a_{6}\right)\right)+\left(a_{7}+a_{8} \xi\right) f\left(a_{9}\left(a_{10}-\xi\right)\right) \\
& +\left(a_{11}+a_{12} \xi\right) f\left(a_{13}\left(a_{14}-\xi\right)\right)+\left(a_{15}+a_{16} \xi\right) f\left(a_{17}\left(a_{18}-\xi\right)\right)
\end{aligned}
$$


TABLE I: Neutron star models (for SLy4 EoS) with $V(\phi)=A \phi^{-2}$ for various values of $A$ and $\phi(0)$. For comparison the GR maximal mass for this $\operatorname{EoS}$ is $2.05 M_{\odot}\left(\rho_{c}=2.86 \times 10^{15} \mathrm{~g} / \mathrm{cm}^{3}, R=9.98 \mathrm{~km}\right)$.

\begin{tabular}{l}
\hline$A=0.005$ \\
\begin{tabular}{|c|c|c|c|c|c|c|c||c|c|c|c|}
\hline$\phi(0)$ & $M_{\max }$, & $\begin{array}{c}R, \\
M_{\odot}\end{array}$ & $\begin{array}{c}\rho_{c}, \\
\mathrm{~km}\end{array}$ & $\begin{array}{c}10^{15} \mathrm{~g} / \mathrm{cm}^{3} \\
\phi(0)\end{array}$ & $\begin{array}{c}M_{\max }, \\
M_{\odot}\end{array}$ & $\begin{array}{c}R, \\
\mathrm{~km}\end{array}$ & $\begin{array}{c}\rho_{c}, \\
10^{15} \mathrm{~g} / \mathrm{cm}^{3}\end{array}$ & $\begin{array}{c}\phi(0) \\
M_{\max }, \\
M_{\odot}\end{array}$ & $\begin{array}{c}R, \\
\mathrm{~km}\end{array}$ & $\begin{array}{c}\rho_{c} \\
10^{15} \mathrm{~g} / \mathrm{cm}^{3}\end{array}$ \\
\hline 0.10 & 2.08 & 10.09 & 2.75 & 0.1 & 2.11 & 10.20 & 2.45 & 0.5 & 2.11 & 10.16 & 2.45 \\
0.05 & 2.09 & 10.15 & 2.64 & 0.05 & 2.13 & 10.41 & 2.26 & 0.25 & 2.14 & 10.35 & 2.26 \\
0.01 & 2.11 & 10.33 & 2.35 & 0.01 & 2.19 & 10.57 & 1.86 & 0.05 & 2.24 & 10.87 & 1.65 \\
\hline
\end{tabular}
\end{tabular}
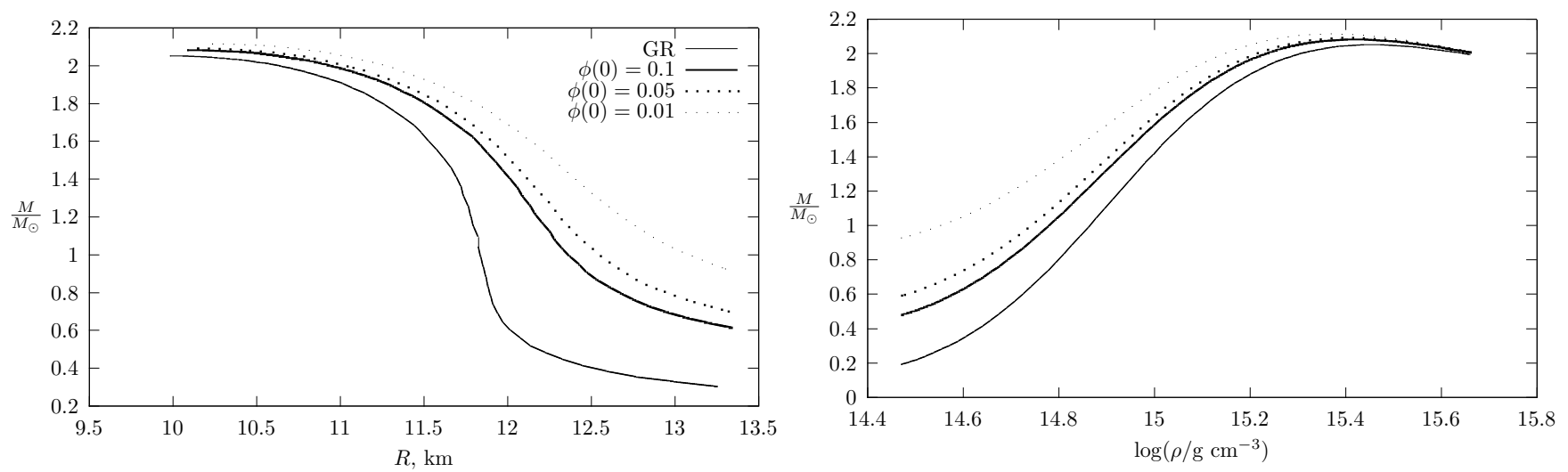

FIG. 1: The mass-radius diagram (left panel) and dependence of neutron star mass on the central density (right panel) for neutron stars in mimetic GR with $V(\phi)=A \phi^{-2}(A=0.005)$ in comparison with GR by using a SLy4 equation of state for various values of $\phi(0)$.

where

$$
\zeta=\log \left(P / \text { dyncm }^{-2}\right), \quad \xi=\log \left(\rho / \mathrm{gcm}^{-3}\right), \quad f(x)=\frac{1}{\exp (x)+1}
$$

and $a_{i}$ are some coefficients.

Let us consider the case of potential $V(\phi)=A \phi^{-2}$. Note that $V\left(\phi^{*}\right)=-A \phi^{*-2}=A^{*} \phi^{*-2}\left(A^{*}=-A\right)$ and $V^{\prime}(\phi)=2 i A^{*} \phi^{*-3}$. From Eq. (10) we obtain equation for real field $\phi^{*}$ :

$$
2 \nabla^{\mu}\left(\beta \partial_{\mu} \phi^{*}\right)+2 A^{*} \phi^{*-3}=0 .
$$

In the rest of the work we omit the asterisk for simplicity. For positive (negative) values of $\phi$ in the center of star one needs to choose the positive (negative) root in Eq. (7) for $d \phi / d r$ (in the opposite case singularity occurs because scalar field $\phi \rightarrow 0)$. Without loss of generality one may assume that $\phi(r)>0$.

The mass-radius (M-R) relation depends on the choice of the value for the scalar mimetic field in center of star, $\phi(0)$. Thus the M-R curves are parametrized by $\phi(0)$, while we assume $\beta(0)=0$. For $A>0$ the mass of star for given radius increases with decrease of $\phi(0)$. The results of calculations are given in Table I. In Fig. 1 the mass-radius diagram and the dependence of the stellar mass on the central density are presented for $A=0.005$ and three values of $\phi(0)$. It is noticeable that the mass-radius relation differs significantly from the corresponding in GR for small masses i.e. $M<\sim 1.4 M_{\odot}$. Also in the low-density regime $\left(\rho_{c}<4 \times 10^{14} \mathrm{~g} / \mathrm{cm}^{3}\right)$ the mimetic gravity models acquire significantly larger masses than GR ones due to scalar contribution.

For the case of exponential potential $V(\phi)=A e^{C \phi^{2}}$ the maximal mass is determined mainly by value of parameter $A$ and weakly depends from $\phi(0)$ (see Table II and Fig. 2).

The presence of the scalar field affects the maximal mass limit. Still the effect is not of the same scale as it has been found for the lower density neutron stars. The effect of the auxiliary field can be more significant for stiffer equations of state (smaller compactness) or/and in the presence of rotation.

The ambiguity of mass-radius relation is determined by the free parameter $\phi(0)$ which can explain some inconsistencies in $M-R$ relation from observations and theoretical considerations. For example, from the study of longer X-ray bursters [23, 24] it follows that neutron stars with masses $M \sim 1-1.4 M_{\odot}$ have large radii $R>14.0 \mathrm{~km}$. However, the analysis of both transiently accreting and bursting sources [25] suggests that the radius of a $1.4 M_{\odot}$ neutron star 
TABLE II: Neutron star models for SLy4 EoS with $V(\phi)=A e^{C \phi^{2}}$ for various values of $A$ and $\phi(0)(C=-0.5)$.

\begin{tabular}{|c||c|c|c|c|c|c|}
\hline \multicolumn{1}{c}{$A=0.02$} & $A=0.04$ \\
\hline$\phi(0)$ & $\begin{array}{c}M_{\text {max }}, \\
M_{\odot}\end{array}$ & $\begin{array}{c}R, \\
\mathrm{~km}\end{array}$ & $\begin{array}{c}\rho_{c}, \\
10^{15} \mathrm{~g} / \mathrm{cm}^{3}\end{array}$ & $\begin{array}{c}M_{\max }, \\
M_{\odot}\end{array}$ & $\begin{array}{c}R, \\
\mathrm{~km}\end{array}$ & $\begin{array}{c}\rho_{c}, \\
10^{15} \mathrm{~g} / \mathrm{cm}^{3}\end{array}$ \\
\hline 0.10 & 2.08 & 10.12 & 2.64 & 2.12 & 10.32 & 2.35 \\
0.05 & 2.09 & 10.10 & 2.64 & 2.12 & 10.32 & 2.35 \\
0.01 & 2.09 & 10.19 & 2.54 & 2.13 & 10.32 & 2.35 \\
\hline
\end{tabular}
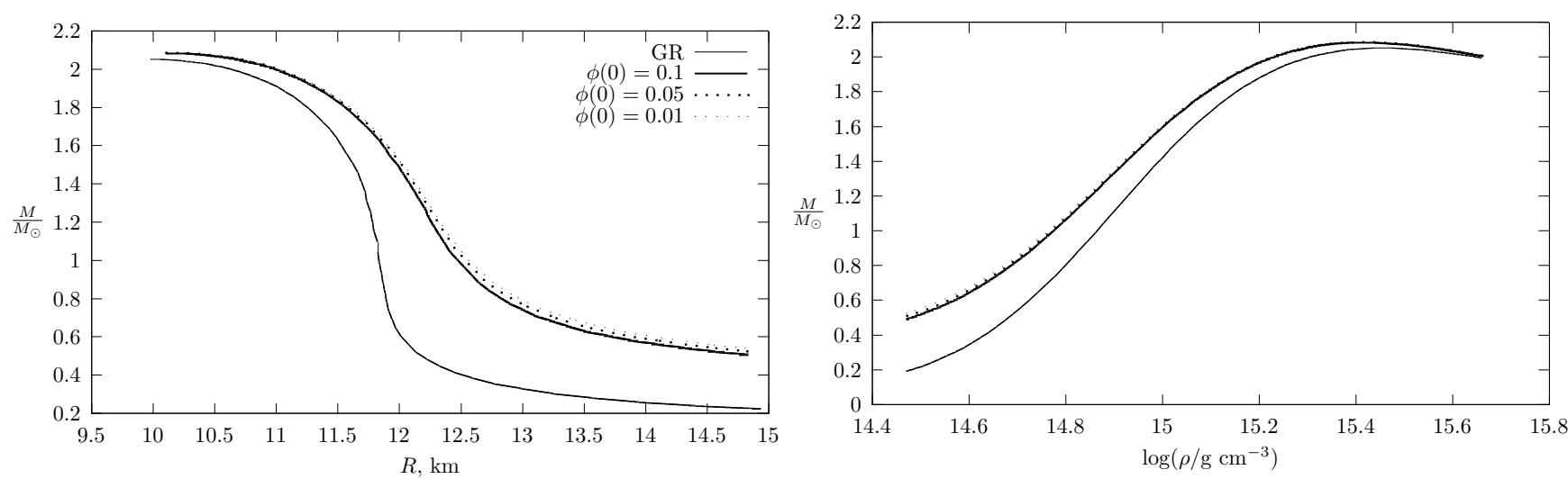

FIG. 2: The mass-radius diagram (left panel) and dependence of neutron star mass on central density (right panel) for neutron stars in mimetic GR with $V(\phi)=A e^{C \phi^{2}}(A=0.02, C=0.5)$ in comparison with GR by using the SLy4 equation of state for various values of $\phi(0)$.

should not exceed the $12.9 \mathrm{~km}$. These contradictions may indicate that the neutron star mass is determined not only by the EOS of the dense matter but also by other parameters. In mimetic gravity these free parameters are present. Of course, there are many other EOS for which neutron stars can have larger radius for the typical $1.4 M_{\odot}$ models. However, our main point is that even EoS which is considered to be not fully realistic due to discovery of large mass neutron mass, may still be viable in modified gravity! The results presented here provide hint that unique discrimination between General Relativity and mimetic gravity can be made once we know in detail the equation of state.

\section{B. Quark stars}

Here we consider the properties of quark stars [26, 27] in mimetic gravity. The hypothetical quark stars consist of deconfined light quarks $(u, d$ and $s$ ) and electrons. The equation for quark matter that forms a colour supeconductor is quite simple in frames of MIT bag model:

$$
p=\alpha(\rho-4 B) .
$$

Here $B$ is the so called "bag constant". The value of parameter $\alpha$ depends on the chosen mass of strange quark and varies from $\alpha=1 / 3\left(m_{s}=0\right)$ to $\alpha=0.28\left(m_{s}=250 \mathrm{MeV}\right)$. The value of $B$ lies in interval $0.98<B<1.52$ in units of $B_{0}=60 \mathrm{MeV} / \mathrm{fm}^{3}$ [30]. It is worth noticing that the discovery of neutron stars with masses of the order of $\approx 2 M_{\odot}$ [28, 29] imposes severe constraints on the equation of state (EOS) of nuclear matter and especially on the possibility of existence of quark stars.

We consider two cases: 1) $\alpha=0.28, B=B_{0}$ and 2) $\alpha=1 / 3, B=B_{0}$ assuming the potential $V(\phi)=A \phi^{-2}$. For quark stars the mass-radius relation is significantly different from the GR one for large masses (see Table III and Fig. 3 for results). The deviation from General Relativity is stronger in comparison with neutron stars (for same $A$ and $\phi(0))$.

For $V(\phi)=A e^{C \phi^{2}}$ the results are similar to above considered for neutron stars. The deviation from General Relativity is defined mainly by value of parameter $A$ (see Fig. 4).

The quark star models under investigation have the maximum mass below the maximum known mass $\approx 2 M_{\odot}$. However, in the framework of mimetic gravity with a simple potential the maximal mass can meet and exceed this 
TABLE III: Quark star models with $V(\phi)=A \phi^{-2}$ for various $A$ and $\phi(0)$. For comparison in General Relativity maximal mass for considered EoS is $1.77 M_{\odot}\left(\rho_{c}=2.26 \times 10^{15} \mathrm{~g} / \mathrm{cm}^{3}, R=10.23 \mathrm{~km}\right)$ for $\alpha=0.28$ and $1.97 M_{\odot}\left(\rho_{c}=2.01 \times 10^{15} \mathrm{~g} / \mathrm{cm}^{3}\right.$, $R=10.76 \mathrm{~km})$ for $\alpha=1 / 3$.

\begin{tabular}{|c|c|c|c|c|c|c|}
\hline \multicolumn{4}{|c|}{$\alpha=0.28$} & \multicolumn{3}{|c|}{$\alpha=1 / 3$} \\
\hline \multicolumn{7}{|c|}{$A=0.005$} \\
\hline$\phi(0)$ & $\begin{array}{c}M_{\max } \\
M_{\odot}\end{array}$ & $\begin{array}{l}R, \\
\mathrm{~km}\end{array}$ & $\begin{array}{c}\rho_{c}, \\
10^{15} \mathrm{~g} / \mathrm{cm}^{3}\end{array}$ & $\begin{array}{c}M_{\max }, \\
M_{\odot}\end{array}$ & $\begin{array}{l}R, \\
\mathrm{~km}\end{array}$ & $\begin{array}{c}\rho_{c}, \\
10^{15} \mathrm{~g} / \mathrm{cm}^{3}\end{array}$ \\
\hline 0.1 & 1.84 & 10.42 & 1.93 & 2.04 & 10.90 & 1.79 \\
\hline 0.05 & 1.86 & 10.50 & 1.79 & 2.06 & 10.99 & 1.65 \\
\hline 0.01 & 1.91 & 10.66 & 1.47 & 2.10 & 11.07 & 1.47 \\
\hline \multicolumn{7}{|c|}{$A=0.01$} \\
\hline 0.1 & 1.91 & 10.62 & 1.65 & 2.11 & 11.01 & 1.53 \\
\hline 0.05 & 1.96 & 10.78 & 1.41 & 2.15 & 11.23 & 1.36 \\
\hline 0.01 & 2.07 & 11.08 & 0.99 & 2.26 & 11.51 & 0.99 \\
\hline
\end{tabular}
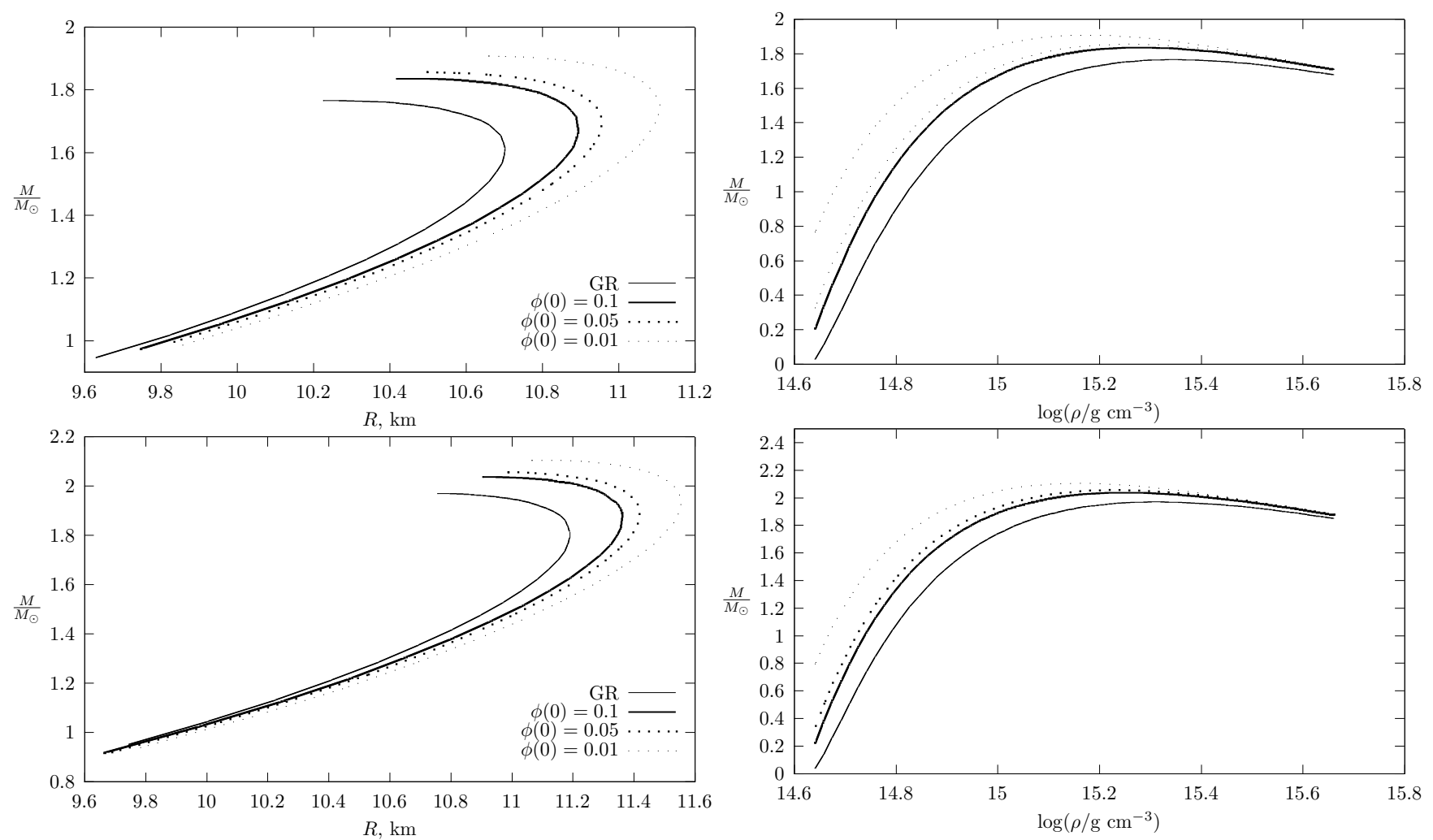

FIG. 3: The mass-radius diagram (left) and the dependence of mass on the central density (right) for quark stars in mimetic GR with $V(\phi)=A \phi^{-2}(A=0.005)$ in comparison with General Relativity. Here we used a simple EoS (28) with $\alpha=0.28$, $B=B_{0}$ (upper panel) and $\alpha=1 / 3, B=B_{0}$ (lower panel) for various values of $\phi(0)$.

limit set by observations.

\section{The inertial characteristics of compact stars in mimetic gravity.}

It is interesting to investigate slowly rotating compact stars in mimetic gravity with potential $V(\phi)=A \phi^{-2}$.

The spacetime metric with only first-order rotational terms with respect to $\Omega=u^{\phi} / u^{t}$ has the form

$$
d s^{2}=-e^{2 \psi(r)} d t^{2}+e^{2 \lambda(r)} d r^{2}+r^{2}\left(d \theta^{2}+\sin ^{2} \theta(d \phi-(\Omega-\omega(r)) d t)^{2}\right) .
$$



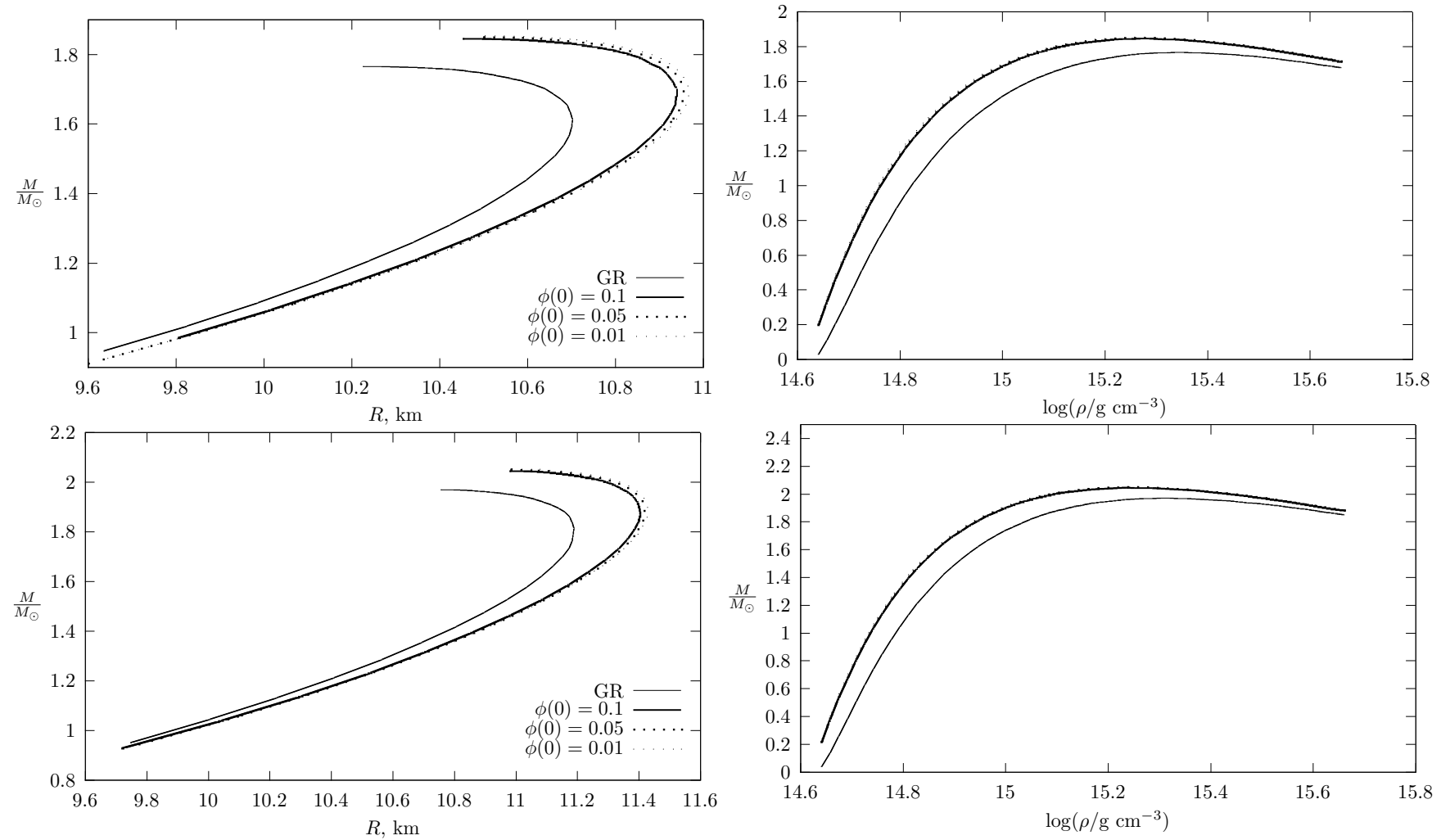

FIG. 4: The mass-radius diagram (left) and dependence of mass on the central density (right) for quark stars in mimetic GR with $V(\phi)=A e^{C \phi^{2}}(A=0.02, C=-0.5)$ in comparison with General Relativity. Here we used the EOS (28) with $\alpha=0.28$, $B=B_{0}$ (upper panel) and $\alpha=1 / 3, B=B_{0}$ (lower panel) for various values of $\phi(0)$. The results seem to be insensitive to the central values of the $\phi(0)$ because the auxiliary field increases quickly and $V(\phi) \rightarrow 0$. In fact the mass-radius relation depends solely on the parameter $A$ and not on $\phi(0)$.

For slowly rotating stars the moment of inertia is given by

$$
I=-\frac{2}{3} \int_{0}^{R} r^{3} \frac{d j}{d r} \frac{\omega(r)}{\Omega},
$$

where $R$ is the radius of non-rotating model and $j(r)=e^{-\lambda(r)-\psi(r)}$.

In the scalar-tensor theory of gravity one needs to add an extra equation for the function $\omega(r)$ to system (20) - (24). Taking only first-order terms on $\omega(r)$, the field equations in the Einstein frame can be written as

$$
\frac{e^{\psi-\lambda}}{r^{4}} \partial_{r}\left[e^{-(\psi+\lambda)} r^{4} \partial_{r} \omega\right]+\frac{1}{r^{2} \sin ^{3} \theta} \partial_{\theta}\left[\sin ^{3} \theta \partial_{\theta} \omega\right]=16 \pi e^{-4 \varphi / \sqrt{3}}(\rho+p) \omega .
$$

In asymptotically flat spacetime $\omega$ is a function of radial coordinate only. Therefore Eq. (31) can be rewritten as

$$
\frac{e^{\psi-\lambda}}{r^{4}} \frac{d}{d r}\left[e^{-(\psi+\lambda)} r^{4} \frac{d \omega(r)}{d r}\right]=16 \pi e^{-4 \varphi / \sqrt{3}}(\rho+p) \omega(r)
$$

The function $\omega(r)$ should obey the following two boundary conditions. Firstly, the condition of regularity at the $r=0$ which requires

$$
\frac{d \omega(0)}{d r}=0
$$

Secondly,

$$
\lim _{r \rightarrow \infty} \omega=\Omega .
$$


TABLE IV: The relative deviations of the maximum mass and the corresponding moment of inertia for neutron stars (SLy4 EoS). The results for moment inertia are given in units $G^{2} M_{\odot}^{3} / c^{4}=4.326 \times 10^{43} \mathrm{~g} \cdot \mathrm{cm}^{2}$.

\begin{tabular}{|c||cccc|cccc|}
\hline \multicolumn{1}{c|}{$\mathrm{A}=0.005$} & \multicolumn{6}{c}{$\mathrm{A}=0.01$} \\
\hline$\phi(0)$ & $M_{\max }$ & $\Delta M_{\max }[\%]$ & $I_{\max }$ & $\Delta I_{\max }[\%]$ & $M_{\max }$ & $\Delta M_{\max }[\%]$ & $I_{\max }$ & $\Delta I_{\max }[\%]$ \\
\hline GR & 2.05 & 0.0 & 46.2 & 0.0 & 2.05 & 0.0 & 46.2 & 0.0 \\
\hline 0.1 & 2.08 & 1.4 & 46.9 & 1.5 & 2.11 & 2.9 & 47.8 & 3.5 \\
0.05 & 2.09 & 2.0 & 47.4 & 2.6 & 2.13 & 3.9 & 49.0 & 6.1 \\
0.01 & 2.11 & 2.9 & 48.8 & 5.6 & 2.19 & 6.8 & 52.5 & 13.0 \\
\hline
\end{tabular}

TABLE V: The relative deviations of the maximum mass and the corresponding moment of inertia for quark stars.

\begin{tabular}{|c|c|c|c|c|c|c|c|c|}
\hline \multicolumn{6}{|c|}{$\alpha=0.28$} & \multicolumn{3}{|c|}{$\alpha=1 / 3$} \\
\hline \multicolumn{9}{|c|}{$\mathrm{A}=0.005$} \\
\hline$\phi(0)$ & $M_{\max }$ & $\Delta M_{\max }[\%]$ & $I_{\max }$ & $\Delta I_{\max }[\%]$ & $M_{\max }$ & $\Delta M_{\max }[\%]$ & $I_{\max }$ & $\Delta I_{\max }[\%]$ \\
\hline GR & 1.77 & 0.0 & 41.3 & 0.0 & 1.97 & 0.0 & 52.3 & 0.0 \\
\hline 0.1 & 1.84 & 4.0 & 43.8 & 6.0 & 2.04 & 3.5 & 54.8 & 4.8 \\
\hline 0.05 & 1.86 & 5.0 & 44.8 & 8.5 & 2.06 & 4.6 & 55.9 & 6.9 \\
\hline 0.01 & 1.91 & 7.9 & 47.5 & 15.0 & 2.10 & 6.6 & 58.7 & 10.9 \\
\hline \multicolumn{9}{|c|}{$\mathrm{A}=0.01$} \\
\hline 0.1 & 1.91 & 7.9 & 46.6 & 12.8 & 2.11 & 7.1 & 57.6 & 10.1 \\
\hline 0.05 & 1.97 & 11.3 & 48.9 & 18.4 & 2.15 & 9.1 & 60.0 & 14.7 \\
\hline 0.01 & 2.07 & 16.9 & 55.5 & 34.4 & 2.26 & 14.7 & 66.9 & 27.9 \\
\hline
\end{tabular}

Then the relation for the moment of inertia can be written as:

$$
I=\frac{8 \pi}{3} \int_{0}^{r_{s}} e^{-4 \varphi / \sqrt{3}}(\rho+p) e^{\lambda-\psi} r^{4}\left(\frac{\omega}{\Omega}\right) d r .
$$

For comparison, we calculate the relative deviation of the maximum mass and the maximum moment of inertia in mimetic gravity and General Relativity:

$$
\Delta M_{\max }=\frac{M_{\max }-M_{\max }^{G R}}{M_{\max }^{G R}}, \quad \Delta I_{\max }=\frac{I_{\max }-I_{\max }^{G R}}{I_{\max }^{G R}} .
$$

The results for neutron and quark stars are given in Tables IV and V correspondingly. The deviations of maximal mass and moment of inertia as function of $\phi_{0}=\phi(0)$ are given on Fig. 5. For given $A$ there is minimal value of $\phi_{0}$ such that for $\phi(0)<\phi_{0}$ stable stars cannot exist.

On Fig. 6 (left panel) the moment of inertia as function of mass is plotted for $A=0.005$ for considered models of neutron and quark stars. For given values of $A$ and $\phi(0)$ the increase of maximal moment of inertia and maximal mass are much stronger than for neutron stars.

We also examined the normalized metric function $\omega(r) / \Omega$ as a function of the radial coordinate. On Fig. 6 this function is depicted for two star configurations with $M=1.4 M_{\odot}$ and mass close to $M_{\max }^{G R}$ for each EoS. One can see that deviations from General Relativity are larger in the vicinity of star core, this is a feature noticed also in 32 ] for slowly rotating neutron star models in $R^{2}$ gravity. The contribution of auxiliary scalar field grows with decreasing $\phi_{0}$ and therefore this leads to stronger deviations from General Relativity.

One may note that the relative increase of the maximum moment of inertia is larger than the increase of the maximal mass and may exceed the EoS uncertainty. Similar trend has been found for neutron star models in scalar tensor theory of gravity and in $R$-squared gravity [14, 32]. Determination of the moment of inertia in double pulsars as assumed can reach high accuracy $(\sim 10 \%)$ see [33]. Therefore these observations can help us to distinguish between General Relativity and its mimetic alternative or set constraints on mimetic potential in the framework of mimetic gravity.

\section{Mimetic $f(R)$ theory}

One should say several words about mimetic $f(R)(f(R) \neq R)$ gravity. In the mathematically equivalent scalartensor theory the potential $V(\varphi)$ can be written in explicit form only for simple $f(R)$ models. For $R$-squared gravity 
a)
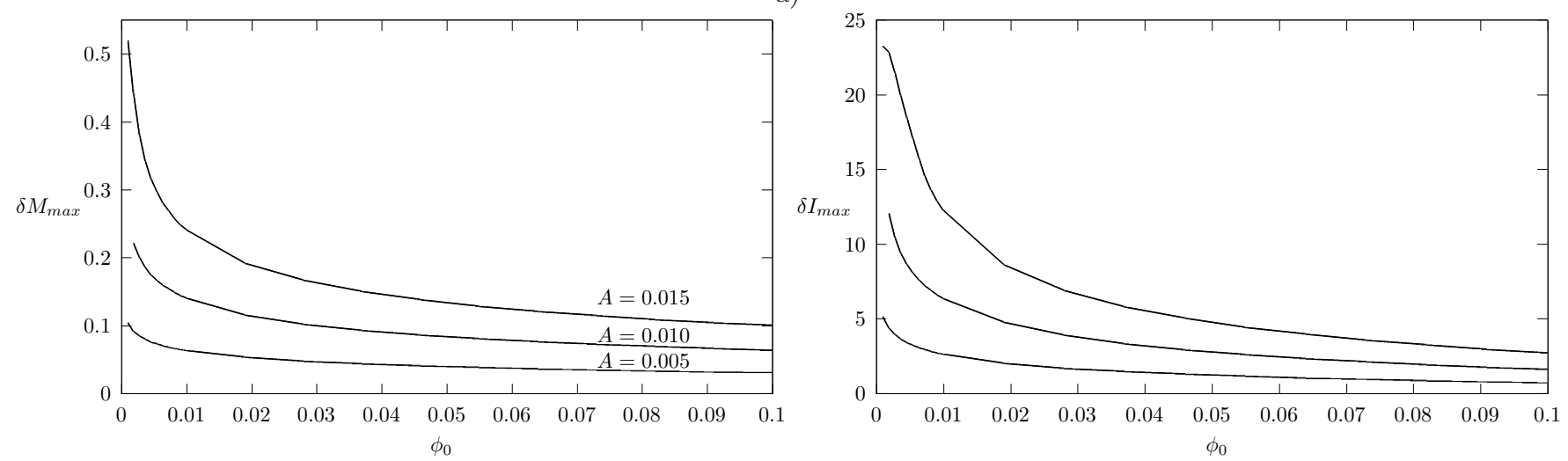

b)
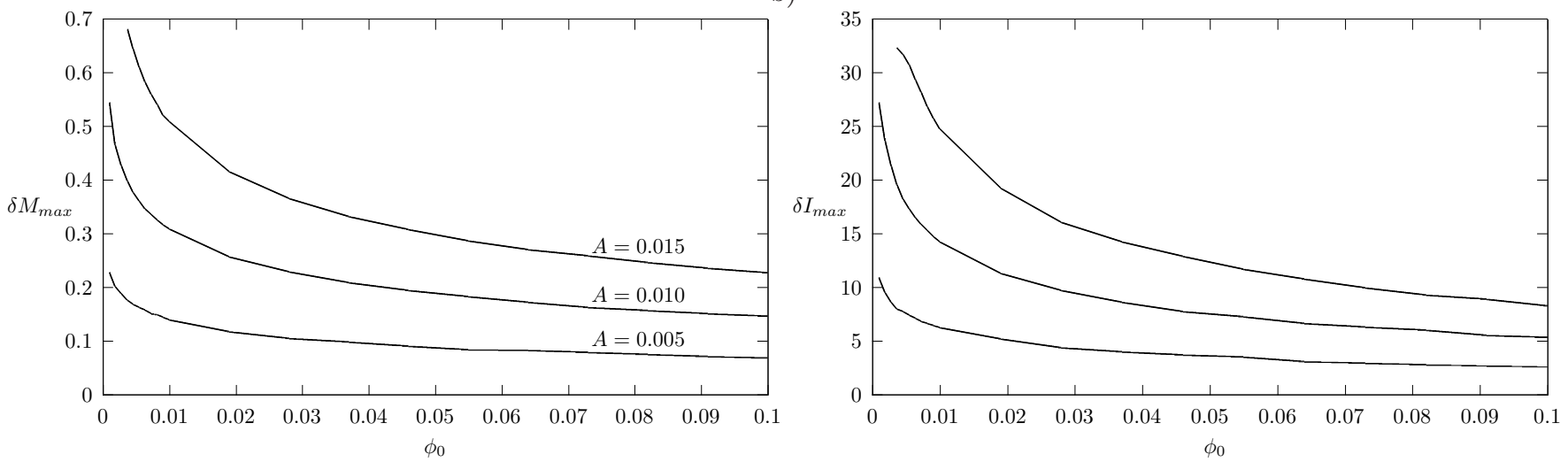

c)
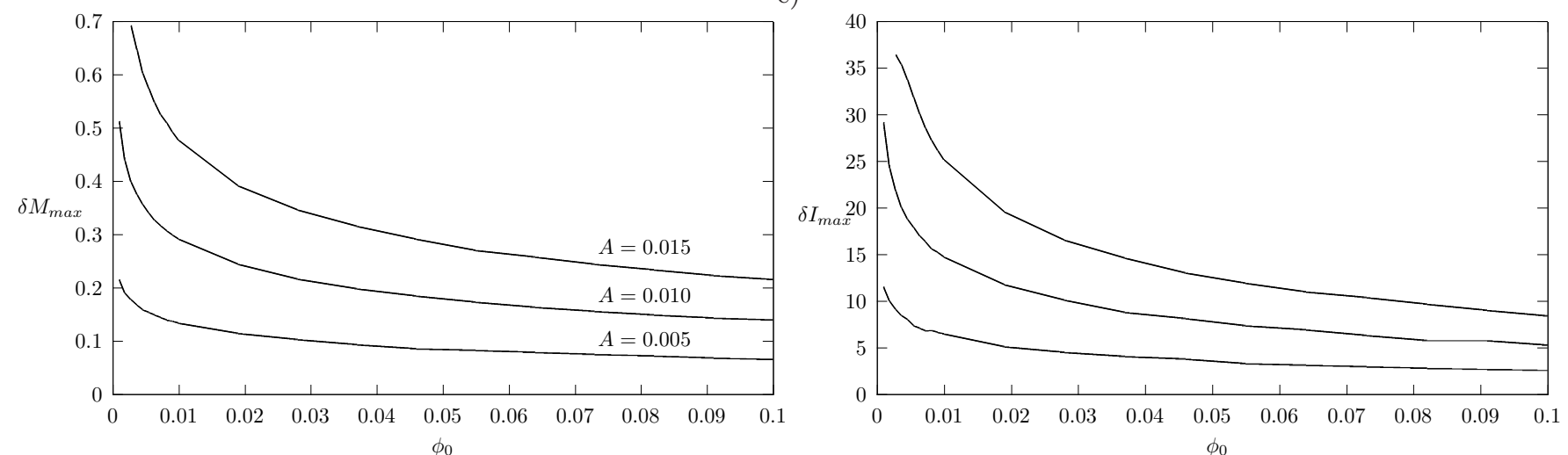

FIG. 5: The deviation of maximal star mass (left panel) and moment of inertia (right panel) from General Relativity as function of $\phi(0)=\phi_{0}$ for some values of parameter $A$ for a) neutron stars using SLy4 EoS, b) quark stars (EoS (28) with $\alpha=0.28$, $\left.B=B_{0}\right)$, c) quark stars $\left(\operatorname{EoS}(28)\right.$ with $\left.\alpha=1 / 3, B=B_{0}\right)$.

$f(R)=R+16 \pi a R^{2}$ one can obtain that

$$
V(\varphi)=\frac{1}{4 a}\left(1-e^{-2 \varphi / \sqrt{3}}\right)^{2} .
$$

The compact star models in non-perturbative $R^{2}$ gravity have been considered in 14, 15, 32, 34. For existence of stable stars one needs the fine-tuning for scalar curvature in the center of star. Only for unique value of scalar curvature in center the solution of TOV equations has required asymptotic at $r \rightarrow \infty$. In terms of scalar-tensor description we have the fine-tuning for scalar field $\varphi$. The gravitational mass increases with increasing $a$. For masses $M<\sim 1.2 M_{\odot}$ (Sly4 EoS) the radii of star configurations decreases with increasing $a$. Considering mimetic theory with scalar potential one can obtain that this decrease can be compensated by contribution of scalar field. Otherwise the increase of maximal mass due to scalar field becomes much stronger in $R^{2}$ gravity. As is shown in [14] the relative 
a)
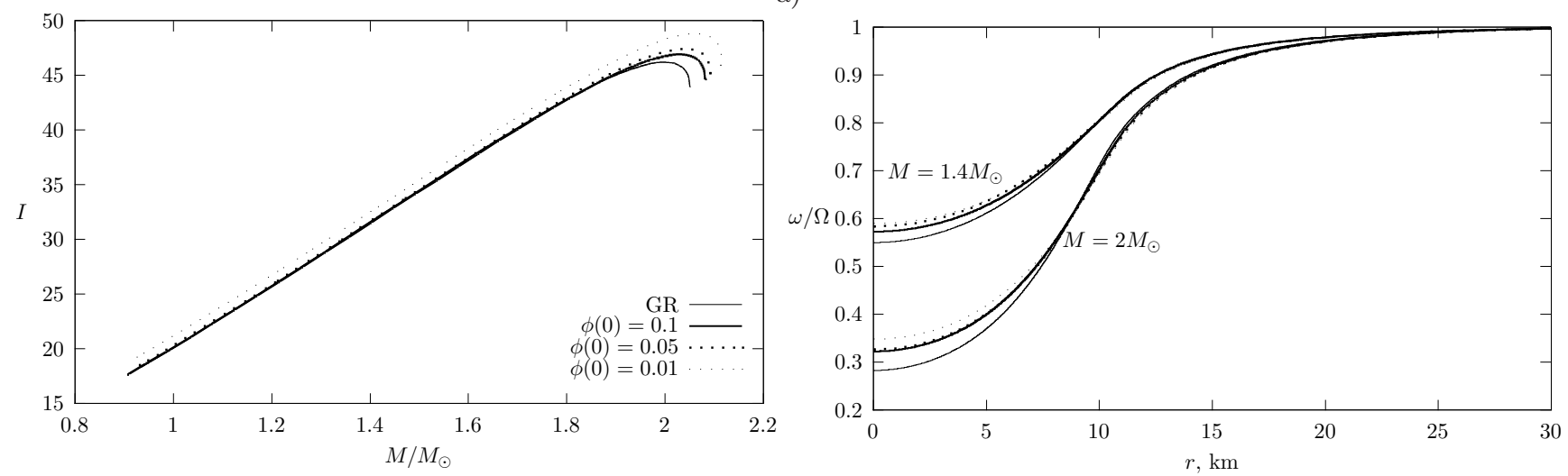

b)
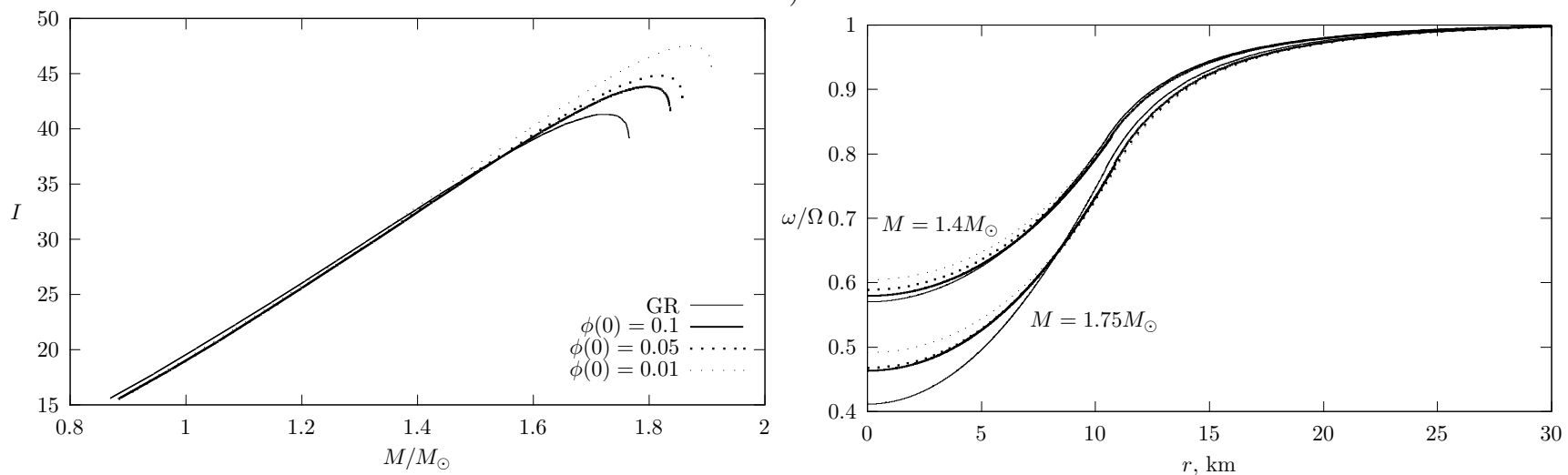

c)
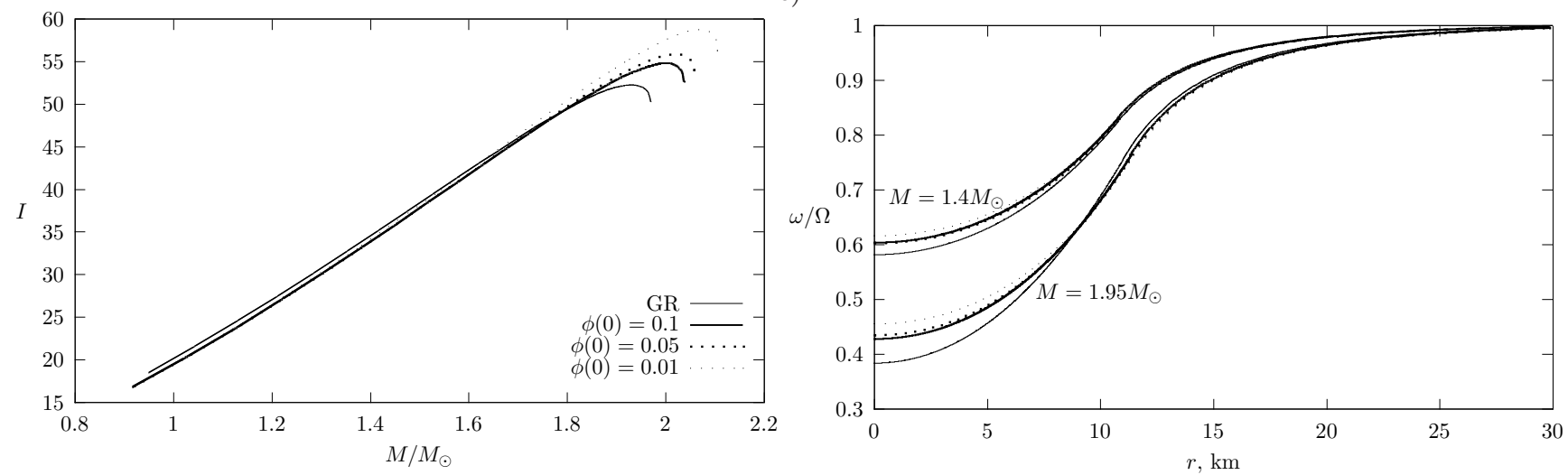

FIG. 6: Left panel: the dependence of quark star moment of inertia from mass in mimetic GR with $V(\phi)=A \phi^{-2}(A=0.005)$ in comparison with General Relativity for a) neutron stars using SLy4 EoS, b) quark stars (EoS (28) with $\alpha=0.28, B=B_{0}$ ), c) quark stars (EoS (28) with $\alpha=1 / 3, B=B_{0}$ ). Right panel: the normalized metric function $\omega(r) / \Omega$ as a function of the radial coordinate for stars with masses $M=1.4 M_{\odot}$ and close to maximal for corresponding EoS.

increase of maximal stellar mass is only $10 \%$ for $R^{2}$ gravity (Einstein frame description). In $F(R)$ frame description it maybe bigger, see [15]. Contribution of scalar field in mimetic gravity can lead to possible existence of extreme neutron stars with large masses.

For quark stars the deviation of mass-radius relation from General Relativity in quadratic gravity is similar to such in mimetic model considered above. Therefore the deviations from General Relativity in vicinity of large masses for $R^{2}$ mimetic gravity can be enhanced with increasing $a$ and $V(\phi)$ in a case of quark stars. This conclusion holds also for inertial characteristic of compact stars. 


\section{CONCLUSION}

In the present paper we considered realistic neutron and quark stars in simple mimetic gravity with mimetic scalar potential. We obtained the mass-radius relations and examined the dependence of inertial characteristics from stellar mass.

For simple potentials of the form $V(\phi)=A \phi^{-2}$ the mass-radius relation for compact stars can considerably deviate from the mass-radius relation in General Relativity. For neutron stars this deviation occurs for stellar configurations with any mass whereas for quark stars the mass-radius relation deviates only for large masses. The deviation from GR depends on the value of mimetic scalar in the center of star. For values of $\phi(0)$ smaller than a specific critical value $\phi_{\text {crit }}$ there exist no stable stellar configurations. The parameter $\phi_{\text {crit }}$ depends on EoS and the form of potential. Due to the contribution of mimetic scalar the maximum mass and the corresponding moment of inertia may increase. This increase is considerably larger for quark stars in comparison with the neutron stars. It should be noted that the presence of mimetic scalar offers the possibility for the existence of stars with low central densities $\rho<10^{15} \mathrm{~g} / \mathrm{cm}^{3}$ but large masses $M>M_{\odot}$.

In mimetic gravity there exists a free parameter (the value of mimetic scalar in the center of the star as initial condition). This freedom leads to ambiguity of mass-radius relation for given equation of state. This ambiguity can potentially explain some contradictions between observations and theoretical modelling of compact stars in General Relativity. The relative deviation of the maximal moment of inertia is approximately two times larger than the relative deviation of maximal stellar mass. Even for negligible increase of mass lying within equation of state uncertainty the increase of moment of inertia (if measured) can help to discriminate between GR and mimetic gravity. Eventually, the future observations of moment of inertia of compact stars will set constraints on the models of mimetic gravity and/or convenient modified gravity.

In case of $f(R)=R+a R^{2}$ mimetic gravity one can expect that the increase of the maximum mass and of the maximum value of the moment of inertia due to the presence of the scalar may become more significant with growth of $a$ in comparison with ordinary $R^{2}$ gravity.

\section{Acknowledgments}

This work is supported in part by project 14-02-31100 (RFBR, Russia) and 2058/60 (MES, Russia) (AVA) and in part by MINECO (Spain), project FIS2013-44881 and I-LINK 1019(SDO). We are grateful to Kostas Kokkotas and Salvatore Capozziello for helpful discussions and useful comments.

[1] S. Capozziello and V. Faraoni, Beyond Einstein Gravity (Springer) New York (2010); S. Capozziello and M. De Laurentis, Phys. Rept.509, 167 (2011) arXiv:1108.6266 gr-qc]; S. Nojiri and S. D. Odintsov, Phys. Rept. 505, 59 (2011) arXiv:1011.0544 [gr-qc]; eConf C 0602061, 06 (2006) [Int. J. Geom. Meth. Mod. Phys. 4, 115 (2007)] hep-th/0601213; A. de la Cruz-Dombriz and D. Saez-Gomez, Entropy 14, 1717 (2012) arXiv:1207.2663 [gr-qc]; T. Clifton, P. G. Ferreira, A. Padilla and C. Skordis, Phys. Rept. 513 (2012) 1 doi:10.1016/j.physrep.2012.01.001 [arXiv:1106.2476] [astro-ph.CO]]

[2] S. Perlmutter et al. [Supernova Cosmology Project Collaboration], Astrophys. J. 517, 565 (1999) arXiv:astro-ph/9812133.

[3] A. G. Riess et al. [Supernova Search Team Collaboration], Astron. J. 116, 1009 (1998) arXiv:astro-ph/9805201.

[4] A. G. Riess et al. [Supernova Search Team Collaboration], Astrophys. J. 607, 665 (2004) arXiv:astro-ph/0402512

[5] D. Psaltis, Living Reviews in Relativity, 11, 9 (2008) arXiv:0806.1531 [astro-ph].

[6] T. Kobayashi and K. i. Maeda, Phys. Rev. D 78, 064019 (2008) arXiv:0807.2503 [astro-ph].

[7] A. Upadhye and W. Hu, Phys. Rev. D 80, 064002 (2009) arXiv:0905.4055 [astro-ph.CO].

[8] D.D. Doneva, S.S. Yazadjiev, K.V. Staykov, K.D. Kokkotas, Phys. Rev. D 90, 104021 (2014), arXiv:1408.1641 [gr-qc]; Phys. Rev. D 92, 064015 (2015).

[9] S. Capozziello, M. De Laurentis, I. De Martino, M. Formisano and S. D. Odintsov, Phys. Rev. D 85, 044022 (2012) arXiv:1112.0761 [gr-qc].

[10] S. Capozziello, M. De Laurentis, S.D. Odintsov and A. Stabile, Phys. Rev. D 83, 064004 (2011) arXiv:1101.0219 [gr-qc].

[11] S. Arapoglu, C. Deliduman, K. Yavuz Eksi, JCAP 1107, 020 (2011) arXiv:1003.3179v3[gr-qc].

[12] H. Alavirad, J.M. Weller, Phys. Rev. D 88, 124034 (2013) arXiv:1307.7977v1[gr-qc].

[13] A. Astashenok, S. Capozziello, S. Odintsov, JCAP 12, 040 (2013) arXiv:1309.1978 [gr-qc]; Phys. Rev. D 89, 103509 (2014) arXiv:1401.4546] [gr-qc]; Astrophys. Space Sci. 355, 333 (2015) arXiv: 1405.6663 [gr-qc]; JCAP 1501, 001 (2015) arXiv:1408.3856 [gr-qc].

[14] S.S. Yazadjiev, D.D. Doneva, K.D. Kokkotas, K.V. Staykov JCAP 1406, 003 (2014); S.S. Yazadjiev, D.D. Doneva, K.D. Kokkotas, Phys. Rev. D 91, 084018 (2015).

[15] S. Capozziello, M. De Laurentis, R. Farinelli and S. D. Odintsov, arXiv:1509.04163 [gr-qc]. 
[16] F. Douchin, P. Haensel Phys. Lett B, 485, 107 (2000)

[17] A.H. Chamseddine and V. Mukhanov, JHEP 1311, 135 (2013) arXiv:1308.5410 [astro-ph.CO]]. A. H. Chamseddine, V. Mukhanov and A. Vikman, JCAP 1406 (2014) 017 doi:10.1088/1475-7516/2014/06/017 arXiv:1403.3961 [astro-ph.CO]]

[18] S. Nojiri and S. D. Odintsov, Mod. Phys. Lett. A 29 (2014) 40, 1450211 doi:10.1142/S0217732314502113 arXiv:1408.3561 [hep-th]]

[19] J. Matsumoto, S. D. Odintsov and S. V. Sushkov, Phys. Rev. D 91 (2015) 6, 064062 doi:10.1103/PhysRevD.91.064062 arXiv:1501.02149 [gr-qc]]; G. Leon and E. N. Saridakis, JCAP 1504 (2015) 04, 031 doi:10.1088/1475-7516/2015/04/031 arXiv:1501.00488 [gr-qc]]; A. V. Astashenok, S. D. Odintsov and V. K. Oikonomou, Class. Quant. Grav. 32 (2015) 18, 185007 doi:10.1088/0264-9381/32/18/185007 arXiv:1504.04861][gr-qc]]; R. Myrzakulov, L. Sebastiani and S. Vagnozzi, Eur. Phys. J. C 75 (2015) 444 doi:10.1140/epjc/s10052-015-3672-6 arXiv:1504.07984 [gr-qc]];D. Momeni, P. H. R. S. Moraes, H. Gholizade and R. Myrzakulov, arXiv:1505.05113 [gr-qc]; M. Raza, K. Myrzakulov, D. Momeni and R. Myrzakulov, doi:10.1007/s10773-015-2891-9 arXiv:1508.00971 [gr-qc]; S. D. Odintsov and V. K. Oikonomou, Annals Phys. 363 (2015) 503 doi:10.1016/j.aop.2015.10.013 [arXiv:1508.07488 [gr-qc]]; arXiv:1511.04559 [gr-qc].

[20] J. Khoury and A. Weltman, Phys. Rev. D 69 (2004) 044026 doi:10.1103/PhysRevD.69.044026 [astro-ph/0309411].

[21] M. Camenzind, Compact Objects in Astrophysics, Springer (2007).

[22] A.Y. Potekhin, A.F. Fantina, N. Chamel, J.M. Pearson, and S. Goriely, arXiv:1310.0049v1 [astro-ph.SR]].

[23] V. Suleimanov, J. Poutanen, M. Revnivstev, and K. Werner, Astrophys. J. 742, 122 (2011).

[24] V. Hambaryan, V. Suleimanov, A. D. Schwope, R. Neuhäuser, K. Werner, and A. Y. Potekhin, Astron. Astrophys. 534, A $74(2011)$.

[25] A.W. Steiner, J.M. Lattimer, E.F. Brown, Astrophys. J. 765, L5 (2013).

[26] N. Itoh, Progress of Theoretical Physics 44, 291 (1970).

[27] E.Witten, Phys. Rev. D 30, 272 (1984).

[28] P.B. Demorest, T. Pennucci, S.M. Ransom, M.S.E. Roberts, J.W.T. Hessels Nature, 467, 1081 (2010).

[29] J. Antoniadis, P.C.C. Freire, N. Wex, T.M. Tauris, R.S. Lynch et.al. Science, 340, 448 (2013).

[30] N. Stergioulas, Living Rev. Rel. 6, 3 (2003) arXiv:gr-qc/0302034 [gr-qc].

[31] J. Naf and P. Jetzer, Phys. Rev. D 81, 104003 (2010) arXiv:1004.2014 [gr-qc].

[32] K.V. Staykov, D.D. Doneva, S.S. Yazadjiev, K.D. Kokkotas, JCAP, 10, 006, (2014).

[33] M. Kramer, N. Wex, Classical and Quantum Gravity 26, 073001 (2009).

[34] A.V. Astashenok, S. Capozziello, S.D. Odintsov, Phys. Lett. B 742, 160 (2015) arXiv:1412.5453 [gr-qc]. 